\title{
Single-molecule fluorescence-based analysis of protein conformation, interaction, and oligomerization in cellular systems
}

\author{
Kenji Okamoto ${ }^{1}$ Michio Hiroshima ${ }^{1,2} \cdot$ Yasushi Sako $^{1}$ \\ Received: 22 September 2017 / Accepted: 19 November 2017 / Published online: 14 December 2017 \\ (C) The Author(s) 2017. This article is an open access publication
}

\begin{abstract}
Single-molecule imaging (SMI) of proteins in operation has a history of intensive investigations over 20 years and is now widely used in various fields of biology and biotechnology. We review the recent advances in SMI of fluorescently-tagged proteins in structural biology, focusing on technical applicability of SMI to the measurements in living cells. Basic technologies and recent applications of SMI in structural biology are introduced. Distinct from other methods in structural biology, SMI directly observes single molecules and single-molecule events one-by-one, thus, explicitly analyzing the distribution of protein structures and the history of protein dynamics. It also allows one to detect single events of protein interaction. One unique feature of SMI is that it is applicable in complicated and heterogeneous environments, including living cells. The numbers, location, movements, interaction, oligomerization, and conformation of single-protein molecules have been determined using SMI in cellular systems.
\end{abstract}

Keywords Fluorescence resonance energy transfer (FRET) $\cdot$ Reaction kinetics $\cdot$ Single-molecule imaging (SMI) · Single-photon counting (SPC) $\cdot$ Super-resolution microscope

\section{Introduction}

Single-molecule imaging (SMI; Sako et al. 2012; Iino et al. 2017) of proteins working in aqueous conditions was first reported in 1995 in the detection of ATP hydrolysis (Funatsu et al. 1995) and sliding movements (Sase et al. 1995) of the muscle actomyosin system. Shortly afterward, SMI was extended to the analysis of Förster resonance energy transfer between single pairs of fluorophore-labeled single-protein molecules (single-pair fluorescence resonance energy transfer; spFRET). spFRET was first used to determine the conformational dynamics of an enzymatic protein (Ha et al. 1999a). Thus, SMI was developed as a technique to study the functional and structural dynamics of protein molecules in vitro.

This article is part of a Special Issue on 'Biomolecules to Bionanomachines - Fumio Arisaka 70th Birthday' edited by Damien Hall, Junichi Takagi and Haruki Nakamura.

Yasushi Sako

sako@ riken.jp

1 Cellular Informatics Laboratory, RIKEN, 2-1 Hirosawa, Wako 351-0198, Japan

2 Laboratory for Cell Signaling Dynamics, QBiC, RIKEN, 6-2-3, Fruedai, Suita 565-0874, Japan
With fluorescence optical microscopes, SMI is easily applicable to complicated biological reaction systems. Observations of fluorophores that are conjugated to single molecules in living cells were reported in 2000 (Sako et al. 2000; Shütz et al. 2000). Biological systems are highly complicated in structure and function. They are working with small numbers of reaction and low energy consumption. Single-molecule resolution is useful to study the mechanism of such biological systems.

SMI provides several types of information on the static and dynamic structure of proteins and other biological macromolecules in vitro and in cellula. spFRET between fluorophores that conjugated to different sites in a single-protein molecule (intra-molecular spFRET) is often used to detect the conformational transition or fluctuation in single molecules of proteins (Ha et al. 1999a; Kozuka et al. 2006; Hibino et al. 2009). In contrast, spFRET between fluorophores that are conjugated to disparate protein molecules (inter-molecular spFRET) allows one to detect the homo- or hetero-oligomerization and interaction of proteins (Sako et al. 2000; Nguyen et al. 2003). Colocalization analysis of multiple molecules is also used to measure protein oligomerization and interaction (Ulbrich and Isacoff 2007; Hiroshima et al. 2012). Although spFRET analysis has higher spatial resolution than colocalization analysis, occurrence of FRET depends on distance and orientation of 
the fluorophores which are difficult to control ad libitum. Fluorescence correlation spectroscopy (FCS), fluorescence cross-correlation spectroscopy (FCCS), and photon counting histogram $(\mathrm{PCH})$ are not imaging but single-molecule detection technologies that generate structural information on proteins (Sako et al. 2012). Detecting the fluctuation of the fluorescence intensity caused by exchange of fluorophores in a fixed focus volume, FCS, FCCS, and PCH determine the lateral diffusion coefficient, which is a function of the effective radius, affinity between two colors of particles, and average and distribution of oligomerization size, respectively. These parameters reflect protein conformation and formation of protein complexes. FCCS and PCH setups are applicable for measuring FRET.

Based on optical microscopy, the spatial resolution of SMI and other single-molecule measurements is limited to several hundreds of nanometers. Even in FRET measurements, the typical detection length between fluorophores is $5 \mathrm{~nm}$. Therefore, information about the distributions and dynamics in nanometer to sub-micrometer structures is obtained primarily by single-molecule measurements. In addition to its low spatial resolution, SMI suffers from several technical difficulties. A primordial problem is its requirement of fluorescence labeling which can deform target molecules. Control experiments using conventional biochemical methods are needed to verify the consistency between results with and without labeling. Instability in fluorescence emission and photobleaching of the fluorophores are the major factors limiting spatial accuracy, temporal resolution, and observation period. Data processing in SMI measurements is often time-consuming.

Despite these drawbacks, SMI provides several types of structural information difficult to obtain using other methods. The assessment of the structural distribution of single species of molecules and the dynamic history of an individual molecule are among the many possibilities with SMI measurements. The structure of proteins might not be constrained to a single conformation. Using NMR measurement, multiple conformations have been suggested for even small proteins including the RAS family GTPases (Ito et al. 1997). Based on recent data using in-cell NMR technology, a significant fraction of proteins in cells has been proposed to be unfolded (Inomata et al. 2009). Post-translational modification, such as phosphorylation and methylation, can cause significant conformational changes in proteins. As a simpler case of structural variance, the clustering number of proteins must assume a distribution. The detection of the structural distribution of proteins is important because protein function and activity are generally specific to a structure.

Moreover, single-protein molecules might shuttle between different conformations over time. It is possible that the conformational drifting is not a simple Markov process ( $\mathrm{Lu}$ et al. 1998; Edman and Riglar 2000; Morimatsu et al. 2007). Tracing the history of single-molecule structures and reactions is indispensable for detecting such complex dynamics. One useful feature of SMI is that it does not require synchronization with regard to measuring kinetics. At equilibrium or in the steady state, the fraction of each conformation does not change globally with time, rendering kinetic measurement in ensemble of molecules difficult. However, each molecule undergoes this conformational transition continually, which can be detected using SMI. This property of SMI is good for tracking non-Markov processes that require statistical analysis in the steady state. In SMI measurements, reactions of different molecules can be synchronized virtually by superimposing the starting points of individual reaction events, then we can analyze the lifetime distribution of each state.

The synchronization of multiple molecules, which is performed for kinetic or dynamic measurements of ensemble molecules, is usually difficult in living cells and in later stages of multi-step reaction systems, but these systems are amenable to asynchronous kinetic measurements in SMI. The function of proteins in living cells can be determined from movements, spatial distribution, and association/dissociation kinetics measured using SMI. Because these observations do not disturb structural measurements that are based on spFRET or fluorescence intensity, we can obtain detailed information on function-structure relationships by using SMI, even in complicated systems, such as living cells. In the following sections, we introduce several recent application of SMI in studies on protein structure in cellular systems. Readers may look up the recent special issues on SMI for further information (Orrit et al. 2014; Biteen and Willets 2017).

\section{Single-molecule FRET measurement}

FRET is extensively used to investigate the structure of biomolecules. FRET occurs between two fluorescent dyes (fluorophores) that are in close proximity. When the donor dye is photo-excited, part of its energy is nonradiatively transferred to the acceptor dye, which consumes it to emit a fluorescence photon. The energy transfer efficiency $E_{\text {FRET }}$ varies with the sixth power of interdye distance $r$ expressed as $E_{F R E T}=\frac{1}{1+\left(r / R_{0}\right)}$, where $R_{0}$ is the Förster distance, at which $E_{\mathrm{FRET}}=0.5$, and which changes drastically in the singlenanometer region. $E_{F R E T}$ also depends on orientation between two dyes; however, in many cases, rotation of dyes during the measurement allows averaging of the orientation factor. $E_{\mathrm{FRET}}$ can be measured experimentally as the ratio of fluorescence intensities of two dyes. When two dyes are conjugated to a single protein, the intramolecular FRET depends on the structure of the labeled protein and varies depending on its conformational change. In particular, spFRET measurements enable one to investigate the conformational dynamics of individual molecules. 


\section{Structural measurements using in-solution spFRET}

One typical measurement using spFRET is the detection of fluorescence from single molecules that are diffusing in solution. When excitation light is focused into solution, only molecules in the focus emit fluorescence (Fig. 1a). If the concentration is sufficiently low such that the average number of molecules in the focus is less than one, fluorescence from a single molecule is detected. For this type of experiments, a confocal microscope is used, usually with single-photon counting (SPC) detectors, such as photomultiplier tubes and avalanche photodiodes. Because a single molecule stays in focus only for a short period, typically a few milliseconds in an aqueous solution, it is detected as a burst of fluorescence photons (Fig. 1b). If the molecules are labeled with a pair of FRET dyes, $E_{\text {FRET }}$ can be calculated for each molecule from the fluorescence photon counts of the two dyes in each burst.

In-solution spFRET measurements can be used to capture the structural distribution of a protein ( $\mathrm{PCH}$ of $E_{F R E T}$; Fig. 1c; Fries et al. 1998; Deniz et al. 1999; Dahan et al. 1999). Different structural species form disparate peaks in a singlemolecule $E_{\mathrm{FRET}}$ histogram. Although $E_{\mathrm{FRET}}$ in solution can be measured in bulk, it merely yields an average of the ensemble, i.e. one cannot distinguish whether all molecules have the same $E_{\mathrm{FRET}}$ or molecules with different $E_{\mathrm{FRET}}$ values are mixed. If the molecular structure changes within a burst of an spFRET measurement, $E_{\mathrm{FRET}}$ is detected between peaks of single structures. Therefore, the shape of the histogram depends on the relative rate of conformational dynamics against the burst duration (Schuler et al. 2001; Gopich and Szabo 2007; Tomov et al. 2012).

Advanced techniques, such as alternative laser excitation (ALEX; Kapanidis et al. 2004) have been developed. ALEX uses two colors of excitation lights, which alternately excite the donor and acceptor at high frequency, such that four types of photon counts, with two excitation colors and two detection colors, are detected separately. Then, in addition to $E_{\mathrm{FRET}}$, the quantity of stoichiometry $S$, which is defined as the labeling ratio between the donor and acceptor on single molecules, is calculated for each burst. Information on $S$ enables one to distinguish bursts with a partially photobleached dye pair and exclude it from the statistics.

ALEX has also been used to examine the oligomerization state of proteins. For example, the ubiquitous recombination mediator RecR in D. radiodurans (drRecR) is thought to form stable homodimers and may form homotetramers. Kim et al. (2012) prepared drRecR monomers that were fluorescently labeled with a donor or acceptor to the artificially induced cysteine at the position of Q133 (Q133C) and found using ALEX that drRecR only formed dimers, not tetramers, in a mixed solution. They also observed that the $\mathrm{N}$-terminus of the drRecR monomer is its dimerization interface, by comparing the ALEX results of drRecR with different labeling positions (Q133C and R37C), and that drRecR forms tetramers only in the presence of the $\mathrm{drRecO}$ protein, which links the $\mathrm{C}$-termini of drRecR homodimers. spFRET measurement at subnanomolar concentration allowed the avoiding of bias from transient interaction in higher concentrations of proteins.

Another advantage of spFRET is the ability to examine the structure of intrinsically disordered proteins (IDPs). Because IDPs are natively unfolded and do not have fixed higher-order structures, their structures are unable to be measured using common methods, such as X-ray crystallography. In-solution spFRET can capture the average $E_{\mathrm{FRET}}$ of a fluctuating structure and reveal, for example, its dependence on the concentration of the denaturant or ionic strength of the solvent (MüllerSpäth et al. 2010; Milles et al. 2012; Soranno et al. 2014;
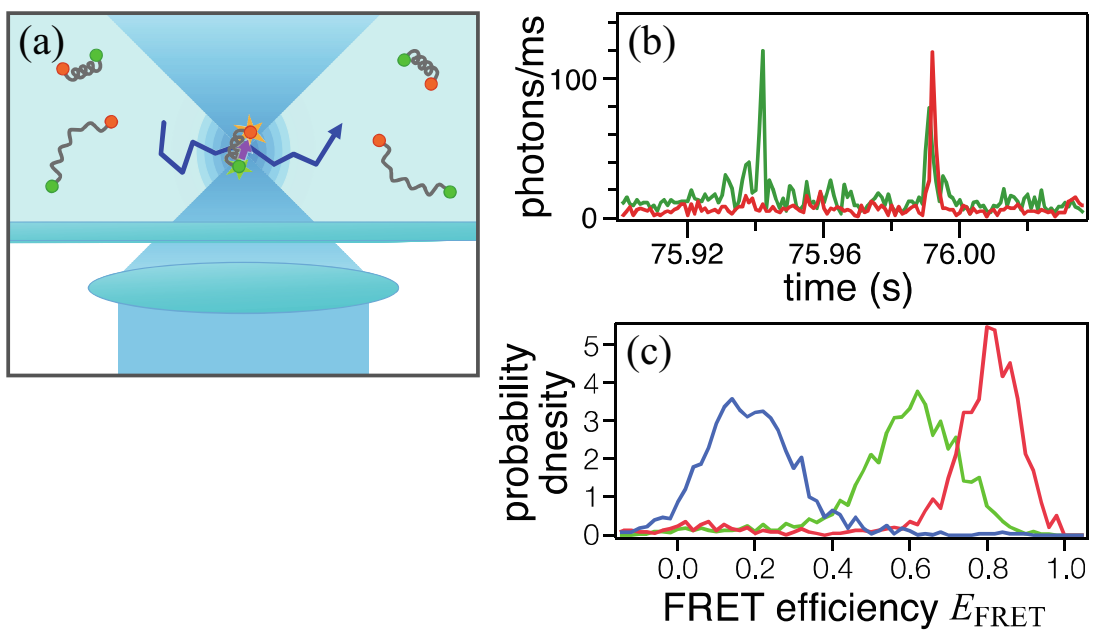

Fig. 1 a Principle of in-solution spFRET measurement. Fluorescence is detected only from a single molecule diffusing through the microscope focus. b Example of fluorescence signals for donor (green) and acceptor (red). Single molecules are detected as bursts of fluorescence. c Example

of spFRET histogram reconstructed from burst signals. Double-helix DNA labeled with interdye distance of 4 (red), 14 (green) and 24 (blue) base-pairs form single peaks (photobleached molecules are excluded by ALEX technique) 
Wuttke et al. 2014). Application to in-cell measurement has also been reported (König et al. 2015), constituting a promising use for spFRET as achieved for FC(C)S (Pack et al. 2014).

\section{spFRET imaging of protein structures in cells}

SMI using high-sensitivity cameras is more common than the SPC detection used in the spFRET measurement in solution. spFRET can be detected simultaneously from multiple molecules by using dual-color SMI. Because diffusing molecules in liquid move too rapidly to image, spFRET imaging and other SMI experiments are typically applied for in vitro systems in which sample molecules are immobilized on a glass substrate surface (Dickson et al. 1997; Lu et al. 1998; Ha et al. 1999b, 2002; Boukobza et al. 2001). spFRET imaging was also successful in live cells (Sako et al. 2000; Murakoshi et al. 2004; Sakon and Weninger 2010). Hibino et al. (2009) performed spFRET imaging of the cytoplasmic serine/threonine kinase RAF on the plasma membrane of cells. RAF is an effector of the membrane protein RAS. RAS is inactive in its GDP-bound form (RAS-GDP) and becomes activated through GDP/GTP exchange. The active GTP-bound form of RAS (RAS-GTP) interacts with downstream effectors, including RAF. With RAF which was genetically labeled with green fluorescent protein (GFP) and yellow fluorescent protein, Hibino et al. observed separate signals from those of single RAF molecules that were bound to RAS on the plasma membrane of living HeLa cells. A difference in $E_{\mathrm{FRET}}$ values obtained before and after cell stimulation with epidermal growth factor (EGF) was detected, indicating that RAF interacts with the inactive RAS-GDP and active RAS-GTP in the high- $E_{\mathrm{FRET}}$ (closed) and low- $E_{\mathrm{FRET}}$ (open) form, respectively.

\section{Time series analysis}

spFRET measurements can trace the dynamics of individual molecules and allow one to analyze, for example, the paths or rates of conformational changes. To do so, spFRET time series are obtained. In SMI measurements of spFRET, $E_{F R E T}$ time series of single molecules are reconstructed from the brightness of fluorescence spots in each frame of a dual-color movie of donor and acceptor channels. Because many single molecules can be measured simultaneously, high-throughput measurements are possible in SMI. In contrast, because the microscope focus is fixed on the target molecule, only a single molecule can be measured at a time, and the molecule must be immobilized during the measurement based on SPC. Conversely, analysis with SPC is precise, because photon statistics are preserved in SPC signals, e.g., detection time of every photon can be recorded. In both types of measurement, data analysis is important because the signal-to-noise ratio in single-molecule detection is usually low. The hidden Markov model (HMM; McKinney et al. 2006; Bronson et al. 2009; Liu et al. 2010) is powerful in recovering the state transition trajectories from spFRET time series under the assumption of discrete states and a simple Markov process for transitions between them. The HMM has been extended to data analysis in SPC measurements (Okamoto and Sako 2012; Pirchi et al. 2016) and of the single-molecule non-Markov process (Zheng and Brown 2004; Sultana et al. 2013; Xue et al. 2015).

Although various measurement and analysis methods have been developed for spFRET experiments, a common reference sample for measuring spFRET dynamics is the Holliday junction (HJ). The HJ is a four-way junction structure that is composed of four DNA strands and possesses stacked and extended structures (Fig. 2a). The crossing point can move by rearrangement of base-pairs near the junction, termed "branch migration". Because the $\mathrm{HJ}$ is easy to treat due to the stability of DNA, it is suitable as a reference for measuring molecular dynamics. Moreover, the rate of the conformational dynamics of HJ can be controlled by the $\mathrm{Mg}^{2+}$ concentration in solution (Panyutin et al. 1995). Several groups have reported a flipflop motion between two stacked structures, reflecting simple two-state transitions with a large change in $E_{\text {FRET }}$ (McKinney et al. 2006; Pirchi et al. 2016), and others have succeeded in resolving spontaneous branch migration, which is the transition between multiple states with relatively small difference in $E_{\text {FRET }}$ (Fig. 2b; Karymov et al. 2008; Okamoto and Sako 2016).

spFRET time series measurements have been applied to protein systems. One typical target is the folding-unfolding (a)

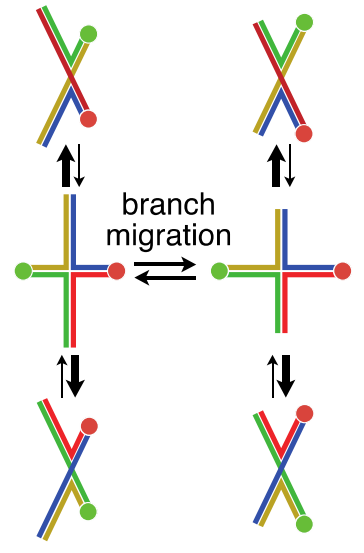

(b)

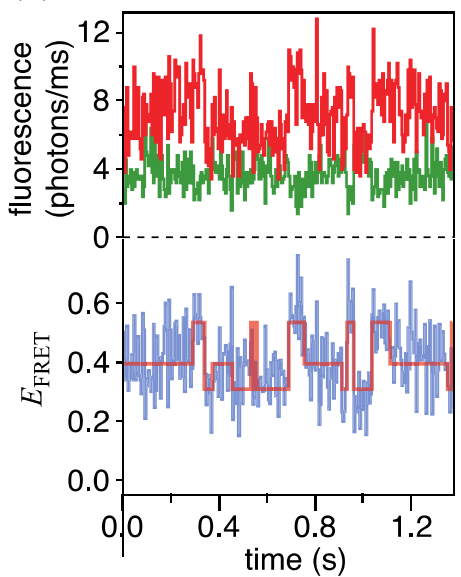

Fig. 2 a The HJ is a four-way junction structure of DNA and assumes stacked (top and bottom) and extended (middle) structures. Branch migration is the movement of the crossing point during transition between extended structures. The green and red circles represent the donor and acceptor dye, respectively. b spFRET measurement results of branch migration between three states. FRET trajectory (blue) was calculated from fluorescence intensities (green for donor, red for acceptor). Red line in the bottom panel is the state transition trajectory obtained by HMM analysis 
dynamics of relatively small proteins. Labeling both ends of a polypeptide with dyes often shows minimal effects on the structure and function of proteins, allowing physiological changes in $E_{\mathrm{FRET}}$ to be detected (Jia et al. 1999; Talaga et al. 2000; Deniz et al. 2000; Schuler and Eaton 2008). spFRET is also used to examine structural dynamics in large complexes, such as ribosomes. Several labeling strategies have been proposed to capture the dynamics of ribosomal conformation and the movements of bound tRNAs in the elongation phase of protein synthesis (Kapp and Lorsch 2004). Ferguson et al. (2015) measured changes in $E_{\mathrm{FRET}}$ over time between fluorescently labeled tRNA substrates that were bound in a human ribosome and found similalities and differences between bacterial and human ribosomes. For example, when a tRNA was bound within the peptidyl $(\mathrm{P})$ site and a second tRNA was introduced to the aminoacyl (A) site, as in bacterial ribosomes, an intermediate-FRET state, in which GTP hydrolysis may take place, appeared after a transient low-FRET codon recognition state before the stable high-FRET bound state. However, the duration of the intermediate-FRET state was longer in human ribosomes, suggesting a distinct mechanism of tRNA selection. Another example is the thermodynamic stability that is observed in the POST complex, in which tRNAs are bound in the P and exit (E) sites, suggesting the existence of factors that are not required for bacterial ribosomes. Such distinct features of human ribosomes compared with bacterial ribosomes indicate a more complex regulation of protein synthesis.

\section{SMI of protein movements and interactions in cells}

SMI allows one to visualize individual and collective molecular behaviors with spatiotemporal resolution in in vitro systems and living cells. Single proteins on the basal plasma membrane of cells that are attached to a glass substrate are observed with a good signal-to-noise ratio using total internal reflection illumination. SMI can even be performed with oblique illumination on the membrane of apical cell surface and cellular organelles, such as the nucleus (Tokunaga et al. 2008). The position and fluorescence intensity of each molecule can be determined in every frame of SMI. The frame-toframe displacement of molecules is converted into the lateral diffusion coefficient or drift velocity, the values of which are often location-specific on the cell surface, reflecting a heterogeneous environment (Marguet et al. 2006). The fluorescence intensity that reflects the number of fluorescent molecules in each spot is used to quantify molecular clustering, which is often important for understanding molecular function (Iino et al. 2001; Ichinose et al. 2004; Ulbrich and Isacoff 2007). When state transitions in the movements or number of molecules are observed, the lifetime distribution of each state involves kinetic information such as the association and dissociation rate constants of molecular interaction (Hiroshima et al. 2012).

\section{Hidden Markov model analysis of protein movements and clustering}

In most cases of SMI, the dynamics of molecular behaviors are analyzed in ensembles of single molecules (Lommerse et al. 2005; Hern et al. 2010). However, HMM, as used in spFRET analysis, is being used increasingly to analyze molecular movements (Chung et al. 2010; Low-Nam et al. 2011; Persson et al. 2013). HMM infers a discrete molecular state for every step in single-molecule trajectories; thus, it determines the real-time transition and real-space distribution of molecular states. HMM assumes that state transition occurs according to the probability matrix, $A_{i j}$, where $i$ and $j$ are the states in the current and next frame, respectively. When local movements of the molecules are considered to be simple lateral diffusion, the distribution of the step displacement for the time interval, $\delta t$, obeys,

$P(r, t)=\sum_{n=1}^{N} \frac{C_{n} r}{2 D_{n} \delta t} \exp \left[\frac{-r^{2}}{4 D_{n} \delta t}\right]$,

where $N$ and $C_{n}$ are the number of states and the fraction of state $n$, respectively. $D_{n}$ is the diffusion coefficient of state $n$, and $r$ is the displacement. The best fit values of these parameters can be estimated, for example, using the expectationmaximization algorithm (Dempster et al. 1977) for each model with a different number of states, and the most suitable number of states is determined by comparing the likelihoods for various state numbers, wherein a penalty for overfitting due to an increase in parameter species (state numbers) is considered. Trajectories of state transitions can be reconstructed, for example, using the Viterbi algorithm (Viterbi 1967).

Early applications of HMM analysis were on the examination of the movement of Q dot-labeled EGF receptor (EGFR), suggesting the existence of three states with different diffusion coefficients and state-specific mobility (Chung et al. 2010; Low-Nam et al. 2011). Each receptor state was believed to reflect movements in specific membrane structure, such as small membrane subdomains. On association of the ligand with EGFR, the diffusion became slower and more confined, possibly due to structural changes in EGFR that were induced by the ligand binding and/or phosphorylation on the cytoplasmic domain. A recent molecular dynamics (MD) simulation suggested a conformational change that could affect the interaction between an EGFR molecule and the membrane surface (Arkhipov et al. 2013).

HMM analysis is also applicable to the transitions in the number of molecules in a protein cluster. Dimerization of 
EGFR is needed for its phosphorylation and subsequent recognition by downstream signaling proteins, and further large oligomerization has also been reported (Clayton et al. 2005; Webb et al. 2008; Huang et al. 2016). Receptor clustering is reflected by an increase in the fluorescence intensity of single spots. To quantify the cluster size, stoichiometric labeling of the target protein is required. To this end, one such method is genetic fusion with a fluorescence tag, such as GFP, and its expression in cells that lack the endogenous protein. The sum of Gaussian distribution can be used as the observation probability in HMM molecular clustering analysis:

$\mathrm{P}(\mathrm{x})=\sum_{n} C_{n} \exp \left[-\frac{(x-n \mu)^{2}}{2 n \sigma^{2}}\right]$.

Here, $x, \mu, \sigma$, and $n$ are the brightness of a spot, brightness of a single molecule, standard deviation for the brightness of single molecules, and cluster size, respectively, and $C_{n}$ is the peak value of the cluster with size $n$. HMM analyses were performed simultaneously on the movements and clustering of EGFR-GFP that was expressed in EGFR-null CHO-K1 cells to identify a moderate correlation that the slowest state tended to be associated with a large cluster (Fig. 3). It is likely that cell signaling by EGFR is regulated by oligomerization beyond dimerization.

\section{SMI of protein interactions}

Single-molecule analysis examines the structural mechanisms that underlie the kinetic properties of protein reactions. In contrast to the conventional kinetic measurements in ensemble molecules, SMI detects the start and end points of individual reaction events directly, and, thus, obtains reaction periods that are not obscured by, for example, the stochasticity that is caused by uncertain wait times for the collision of reactants, rendering SIM measurements suitable for detailed reaction analysis. In addition, because it is based on fluorescence detection, SMI measurement can be made in cellula (Nakamura et al. 2016; Yoshizawa et al. 2017).

The association of a cell differentiation factor, heregulin (HRG), with its receptors (HRGRs) and receptor dimerization were analyzed using SMI in living cells (Hiroshima et al. 2012). HRGRs, which are families of EGFR, form monomers and dimers on the cell surface. The association of a HRG that is labeled with a single fluorophore molecule was observed as the appearance of a fluorescent spot (a stepwise increase in fluorescence intensity) on the cell surface. During sparse ligand binding, the association of two HRG molecules at the same position indicates associations to HRGR dimers. For the first ligand associations to HGFR dimers, the distribution of wait times after the ligand was mixed with the culture medium showed a simple exponential decay. In contrast, the times between the first and second associations showed a distribution with a peak, indicating that an additional rate-limiting step exists prior to the second binding. As with the association, the dissociation of HRG from its receptors was detected as stepwise decreases in fluorescence intensity. The association and dissociation rate constants were obtained by fitting the time distributions using the appropriate chemical reaction models. There were three affinities between HRG and HRGRs. The highest affinity was found with vacant HRGR dimers, but association of the first ligand with vacant dimers decreased the affinity with the second ligand. The affinity with HRGR monomers was the lowest. Depletion of the highest affinity binding sites as the increase of ligand concentration results in a negative cooperativity. A structural change in HRGR dimers during the first and second association with HRG, observed as the additional rate-limiting step, caused negative cooperativity by increasing the dissociation rate constant (Fig. 4).

Extracellular signals to the EGFR family are transmitted into the cytoplasm to induce cell responses. The cytoplasmic proteins that recognize the phosphorylated EGFR family contain Src homology 2 (SH2) domains. Associations between EGFR and SH2 proteins have been investigated by SMI in cells (Jadwin et al. 2016). The temporal dynamics in the recruitment from the cytoplasm to the plasma membrane differ
Fig. 3 Spatial distribution of EGFR states along singlemolecule trajectories for a diffusion and $\mathbf{b}$ clustering. Colors in (a) indicate three diffusion states: immobile (blue), slow diffusion (orange), and fast diffusion $(r e d)$. In (b), cluster sizes from monomer to tetramer are colored from yellow to red. Scale bar $3 \mu \mathrm{m}$ (a)

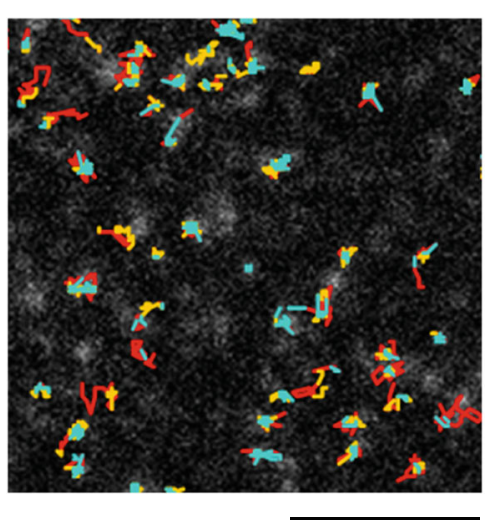

(b)

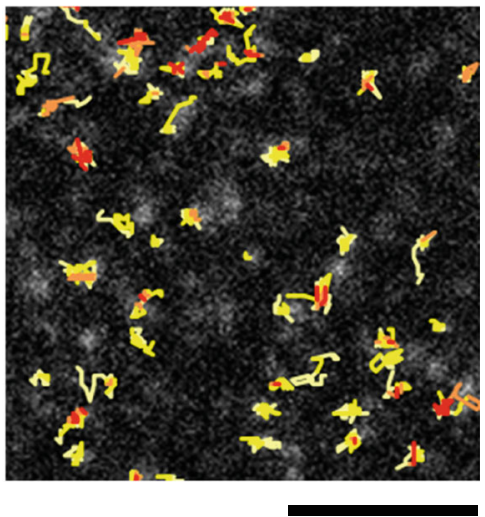


(a)
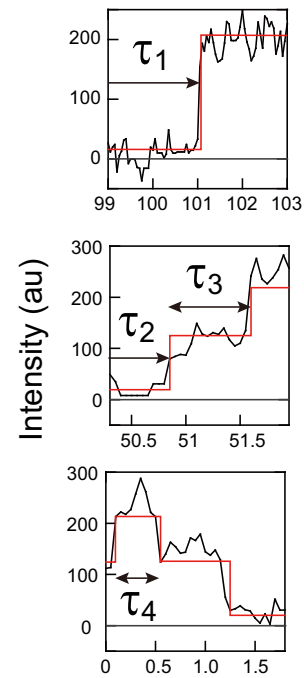

Time (s) (b)
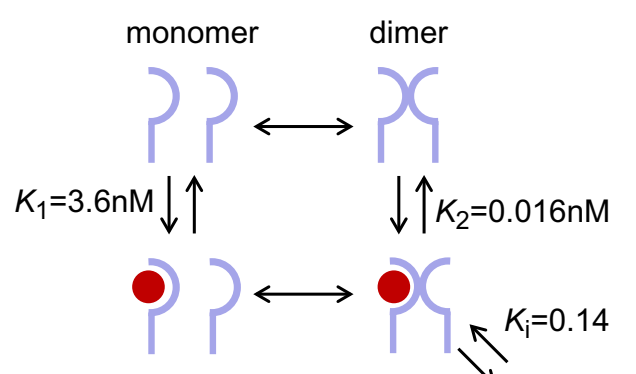

$K_{1}=3.6 \mathrm{nM} \downarrow \uparrow$

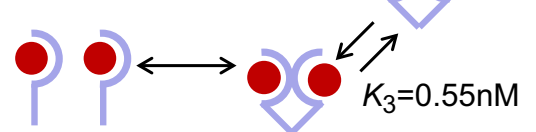

(c)

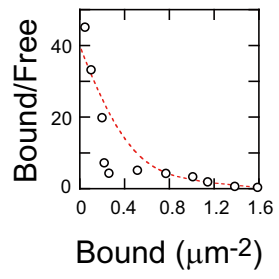

Fig. 4 a Stepwise changes in the brightness of a single fluorescence spot. Thick arrows indicate associations or dissociation of fluorescent HRG. $\tau_{1}-\tau_{3}$ indicate wait times for association of HRG with receptor monomer, vacant receptor dimer, and receptor dimer with one ligand, respectively. $\tau_{4}$ indicates wait time for dissociation from receptor dimer with two ligands. b Reaction scheme of HRG association and receptor

between SH2 proteins in living cells. Among SH2 proteins, GRB2 has a slower dissociation rate constant from EGFR clusters versus monomers. Multiplicity in the reaction rate constants between EGFR and GRB2 has also been observed in in vitro SMI measurement, wherein the association rate constant correlates inversely with the GRB2 concentration (Morimatsu et al. 2007). These results suggest that the cytoplasmic domain of EGFR binds GRB2 in multiple conformations and that the conformation bias depends on the size of the EGFR cluster.

\section{SMI of protein colocalizations and interactions}

In-cell dual-color SMI allows one to compare the dynamics and kinetics between two molecular species. Ichinose et al. (2004) detected single molecules of EGF that were labeled with tetrametylrhodamine (TMR) and an active form of EGFR with Alexa488-labeled antibody to examine the association of EGF and activation of EGFR. Time-lapse dual-color SMI in semi-intact cells revealed that the density and cluster size of active EGFR molecules increased with time, exceeding those of EGF which induced the EGFR activation, meaning that an amplification of the EGF signal occurred through the dynamic EGFR clustering that will cause secondary activation of vacant receptor molecules.

Dynamic colocalization analysis of two protein species detects the kinetics of protein interactions. We have observed the interaction between GFP-tagged EGFR and a cytoplasmic protein dimerization. An intermediate state exists between dimers with one and two ligands. Three dissociation equilibrium constants $K_{1}, K_{2}$, and $K_{3}$, which depend on the receptor states, are indicated. $\mathbf{c}$ Scatchard plot for the association of HRG as an ensemble of molecules shows negative cooperativity. Dotted line was calculated based on the reaction model and parameters in (b)

GRB2 that had been tagged with TMR-labeled Halo protein in living cells. HMM is used to determine the colocalization periods, during which two differently colored spots overlap. The apparent reaction rate constant, as determined from the SMI measurements, must be corrected by the concentrations of labeled and unlabeled GRB2 molecules in the cytoplasm to obtain the true value. These GRB2 concentrations can be determined using FCS and western blot. The affinities with GRB2 differed for monomeric and clustered EGFR molecules, suggesting that a conformational change occurred after molecular clustering.

Another SMI method detects the extent of oligomerization of membrane proteins by counting the number of photo-bleaching steps in single fluorescent spots. GFP molecules that were fused to one subunit, two identical subunits, and two different subunits in a $\mathrm{Ca}^{2+}$ channel, $\mathrm{CNG}$ channel, and NMDA receptor, respectively, were used to measure the clusters in living cells (Ulbrich and Isacoff 2007). The stoichiometry of subunits in the channels/ receptor was reflected by the number of bleaching steps counted. The fraction of non-fluorescent GFP was estimated to be $\sim 20 \%$, assuming binominal distribution of the photobleaching steps. This method can be applied to the quantitative analysis of other proteins with a biologically relevant cluster size and distribution.

\section{Super-resolution microscopy}

Super-resolution microscopy techniques, such as photoactivated localization microscopy and stochastic optical 
reconstruction microscopy, determine the molecular positions with an accuracy of ten to several tens of nanometers, even for condensed molecules, such as those in protein clusters (Betzig et al. 2006; Rust et al. 2006). These techniques use images of momentarily discretized fluorescence emission from single molecules that are labeled with photo-convertible or photo-activable fluorescent probes, including mEOS, mKikGR, PA-mCherry, and PA-GFP (Lukyanov et al. 2005). Using super-resolution microscopy, a ring-like structure that was composed of actinadducin complexes was clearly observed to be winding around axons with a periodicity of $180-190 \mathrm{~nm}$, which is comparable with the size of spectrin tetramers that bridge the rings (Xu et al. 2013). Also, a localized pattern of chemotaxis-related protein clusters was resolved in E.coli cells allowing a mathematical model for chemotactic sensing to be constructed (Greenfield et al. 2009). The arrangement of gp120 in the nuclear pore complex was imaged as an eightfold symmetric structure with a central channel (Löschberger et al. 2012). As shown in these examples, super-resolution microscopy has been used to resolve protein arrangements that were unable to be determined using conventional crystallographic methods.

\section{Conclusion}

Single-molecule fluorescence detection is useful for obtaining structural information on proteins and other biological macromolecules in cellula and in vitro. The greatest advantage of single-molecule measurements is that they provide information on structural distribution and dynamics. Having multiple conformations is a salient property of multi-domain proteins. In addition, single-molecule measurements allow one to perform a structural analysis of proteins and protein complexes without rigid structures, such as IDPs and dynamic protein clusters in cells. Because of often working as important HUB molecules in intracellular reaction networks for signal transduction, gene regulation, and metabolism, the dynamic structure-function relationship of these proteins and protein complexes in living cells is a highly interesting field.

Unfortunately, the structural information that single-molecule measurement provides is limited. Even using spFRET, which has a spatial resolution of $1 \mathrm{~nm}$ or less, only the distance and/ or orientation between two locations on a molecule can be known. No atomic-level three-dimensional structure can be determined directly from single-molecule measurements. Cooperation with other technologies in structural analysis is indispensable. The development of novel methods to maximize the information from single-molecule measurements is ongoing. Data assimilation combining spFRET measurement and MD simulation (Matsunaga et al. 2015) may compensate for the poor spatial resolution in SMI. Multimodal measurements to enrich information, such as the simultaneous detection of polarization and fluorescence lifetime in spFRET measurement (Chung et al.
2016), will improve single-molecule structural biology. In-cell time series measurement with high temporal resolution may be another desirable technology. With the improvements in technologies, the roles of single-molecule measurements in structural biology are really increasing.

\section{Compliance with ethical standards}

Funding This work was supported by MEXT, Japan (JP16H00788, JP15H02394, and JP15KT0087 to YS).

Conflict of interest Kenji Okamoto declares that he has no conflict of interest. Michio Hiroshima declares that he has no conflict of interest. Yasushi Sako declares that he has no conflict of interest.

Ethical approval This article does not contain any studies with human participants or animals performed by any of the authors.

Open Access This article is distributed under the terms of the Creative Commons Attribution 4.0 International License (http:// creativecommons.org/licenses/by/4.0/), which permits unrestricted use, distribution, and reproduction in any medium, provided you give appropriate credit to the original author(s) and the source, provide a link to the Creative Commons license, and indicate if changes were made.

\section{References}

Arkhipov A, Shan Y, Das R, Endres NF, Eastwood MP, Wemmer DE, Kuriyan J, Shaw DE (2013) Architecture and membrane interactions of the EGF receptor. Cell 152:557-569

Betzig E, Patterson GH, Sougrat R, Lindwasser OW, Olenych S, Boni JS (2006) Imaging intracellular fluorescent proteins at nanometer resolution. Science 313:1642-1645

Biteen J, Willets KA (2017) Introduction: super-resolution and singlemolecule imaging. Chem Rev 117:7241-7243

Boukobza E, Sonnenfeld A, Haran G (2001) Immobilization in surfacetethered lipid vesicles as a new tool for single biomolecule spectroscopy. J Phys Chem B 105:12165-12170

Bronson JE, Fei J, Hofman JM, Gonzalez RL Jr, Wiggins CH (2009) Learning rates and states from biophysical time series: a Bayesian approach to model selection and single-molecule FRET data. Biophys J 97:3196-3205

Chung I, Akita R, Vandlen R, Toomre D, Schlessinger J, Mellman I (2010) Spatial control of EGF receptor activation by reversible dimerization on living cells. Nature 464:783-787

Chung HS, Louis JM, Gopich IV (2016) Analysis of fluorescence lifetime and energy transfer efficiency in single-molecule photon trajectories of fast-folding proteins. J Phys Chem B 120:680-699

Clayton AHA, Tavarnesi ML, Johns TG (2005) Unligated epidermal growth factor receptor forms higher order oligomers within microclusters on A431 cells that are sensitive to tyrosine kinase inhibitor binding. Biochemistry 46:4589-4597

Dahan M, Deniz AA, Ha T, Chemla DS, Schultz PG, Weiss S (1999) Ratiometric measurement and identification of single diffusing molecules. Chem Phys 247:85-106

Dempster AP, Laird NM, Rubin DB (1977) Maximum likelihood from incomplete data via the EM algorithm. J R Stat Soc Ser B 39:1-38

Deniz AA, Dahan M, Grunwell JR, Ha T, Faulhaber AE, Chemla DS, Weiss S (1999) Single-pair fluorescence resonance energy transfer on freely diffusing molecules: observation of Förster distance dependence and subpopulations. Proc Natl Acad Sci U S A 96:3670-3675 
Deniz AA, Laurence TA, Beligere GS, Dahan M, Martin AB, Chemla DS, Dawson PE, Schultz PG, Weiss S (2000) Single-molecule protein folding: diffusion fluorescence resonance energy transfer studies of the denaturation of chymotrypsin inhibitor 2. Proc Natl Acad Sci U S A 97:5179-5184

Dickson RM, Cubitt AB, Tsien RY, Moerner WE (1997) On/off blinking and switching behaviour of single molecules of green fluorescent protein. Nature 388:355-358

Edman L, Riglar R (2000) Memory landscapes of single-enzyme molecules. Proc Natl Acad Sci U S A 97:8266-8271

Ferguson A, Wang L, Altman RB, Terry DS, Juette MF, Burnett BJ, Alejo JL, Dass RA, Parks MM, Vincent CT, Blanchard SC (2015) Functional dynamics within the human ribosome regulate the rate of active protein synthesis. Mol Cell 60:475-486

Fries JR, Brand L, Eggeling C, Kollner M, Seidel CAM (1998) Quantitative identification of different single molecules by selective time-resolved confocal fluorescence spectroscopy. J Phys Chem A 102:6601-6613

Funatsu T, Harada Y, Tokunaga M, Saito K, Yanagida T (1995) Imaging of single fluorescent molecules and individual ATP turnovers by single myosin molecules in aqueous solution. Nature 374:555-559

Gopich IV, Szabo A (2007) Single-molecule FRET with diffusion and conformational dynamics. J Phys Chem B 111:12925-12932

Greenfield D, McEvoy AL, Shroff H, Grooks GE, Wingreen NS, Betzig E, Liphardt J (2009) Self-organization of the Escherichia Coli chemotaxis network imaged with super-resolution light microscopy. PLoS Biol 7:e1000137

Ha T, Ting AY, Liang J, Caldwell WB, Deniz AA, Chemla DS, Schultz PG, Weiss S (1999a) Single-molecule fluorescence spectroscopy of enzyme conformational dynamics and cleavage mechanism. Proc Natl Acad Sci U S A 96:893-898

Ha T, Zhuang X, Kim HD, Orr JW, Williamson JR, Chu S (1999b) Ligand-induced conformational changes observed in single RNA molecules. Proc Natl Acad Sci U S A 96:9077-9082

Ha T, Rasnik I, Cheng W, Babcock HP, Gauss GH, Lohman TM, Steven Chu S (2002) Initiation and re-initiation of DNA unwinding by the Escherichia Coli rep helicase. Nature 419:638-641

Hern JA, Baig AH, Mashanov GI et al (2010) Formation and dissociation of M1 muscarinic receptor dimers seen by total internal reflection fluorescence imaging of single molecules. Proc Natl Acad Sci U S A 107:2693-2698

Hibino K, Shibata T, Yanagida T, Sako Y (2009) A RasGTP-induced conformational change in C-RAF is essential for accurate molecular recognition. Biophys J 97:1277-1287

Hiroshima M, Saeki Y, Okada-Hatakeyama M, Sako Y (2012) Dynamically varying interactions between heregulin and ErbB proteins detected by single-molecule analysis in living cells. Proc Natl Acad Sci U S A 109:13984-13989

Huang Y, Bharill S, Karandur D, Peterson SM, Marita M, Shi X, Kaliszewski MJ, Smith AW, Isacoff EY (2016) Molecular basis for multimerization in the activation of the epidermal growth factor receptor. elife 5:1-27

Ichinose J, Murata M, Yanagida T, Sako Y (2004) Single molecule observation of amplification of EGF receptor activation in semi-intact A431 cells. Biochem Biophys Res Commun 324:1143-1149

Iino R, Koyama I, Kusumi A (2001) Single molecule imaging of green fluorescent protein in living cells: E-cadherin form oligomers on the free cell surface. Biophys J 80:2667-2677

Iino R, Iida T, Nakamura A, Saita E, You H, Sako Y (2017) Singlemolecule imaging and manipulation of biomolecular machines and systems. Biochim Biophys Acta. https://doi.org/10.1016/j.bbagen. 2017.08.008 General Subjects. Available online

Inomata $\mathrm{K}$, Ohno A, Tochio H, Isogai S, Tenno T, Nakase I, Takeuchi T, Futaki S, Ito Y, Hiroaki H, Shirakawa M (2009) High-resolution mulit-dimensional NMR spectroscopy of proteins in human cells. Nature 458:106-109
Ito Y, Yamasaki K, Iwahara J, Terada T, Kamiya A, Shirouzu M, Muto Y, Kwai G, Yokayama S, Laue ED, Wälchli M, Shibata T, Nishimura S, Miyazawa T (1997) Regional polysterism in the GTP-bound form of the human c-Ha-Ras protein. Biochemistry 36:9109-9119

Jadwin JA, Oh D, Curran TG, Ogiue-Ikeda M, Jia L, White FM, Machida K, Yu J, Mayer BJ (2016) Time-resolved multimodal analysis of src homology 2 ( $\mathrm{SH} 2)$ domain binding in signaling by receptor tyrosine kinases. elife 5:1-26

Jia Y, Talaga DS, Lau WL, Lu HSM, DeGrado WF, Hochstrasser RM (1999) Folding dynamics of single GCN-4 peptides by fluorescence resonant energy transfer confocal microscopy. Chem Phys 247:69-83

Kapanidis AN, Lee NK, Laurence TA, Doose S, Margeat E, Weiss S (2004) Fluorescence-aided molecule sorting: analysis of structure and interactions by alternating-laser excitation of single molecules. Proc Natl Acad Sci U S A 101:8936-8941

Kapp LD, Lorsch JR (2004) The molecular mechanics of eukaryotic translation. Annu Rev Biochem 73:657-704

Karymov MA, Chinnaraj M, Bogdanov A, Srinivasan AR, Zheng G, Olson WK, Lyubchenko YL (2008) Structure, dynamics, and branch migration of a DNA Holliday junction: a single-molecule fluorescence and modeling study. Biophys J 95:4372-4383

Kim C, Kim JY, Kim SH, Lee BI, Lee NK (2012) Direct characterization of protein oligomers and their quaternary structures by singlemolecule FRET. Chem Commun 48:1138-1140

König I, Zarrine-Afsar A, Aznauryan M, Soranno A, Wunderlich B, Dingfelder F, Stüber JC, Plückthun A, Nettels D, Schuler B (2015) Single-molecule spectroscopy of protein conformational dynamics in live eukaryotic cells. Nat Methods 12:773-779

Kozuka J, Yokota H, Arai Y, Ishii Y, Yanagida T (2006) Dynamic polymorphism of single actin molecules in the actin filament. Nat Chem Biol 2:83-86

Liu Y, Park J, Dahmen KA, Chemla YR, Ha T (2010) A comparative study of multivariate and univariate hidden Markov modelings in time-binned single-molecule FRET data analysis. J Phys Chem B 114:5386-5403

Lommerse PHM, Snaar-Jagalska BE, Spaink HP, Schmidt T (2005) Single-molecule diffusion measurements of H-Ras at the plasma membrane of live cells reveal microdomain localization upon activation. J Cell Sci 118:1799-1809

Löschberger A, van de Linde S, Dabauvalle MC, Rieger B, Heilemann M, Krohne G, Sauer M (2012) Super-resolution imaging visualizes the eightfold symmetry of gp210 proteins around the nuclear pore complex and resolves the central channel with nanometer resolution. J Cell Sci 125:570-575

Low-Nam ST, Lidke KA, Cutler PJ et al (2011) ErbB1 dimerization is promoted by domain co-confinement and stabilized by ligand binding. Nat Struct Mol Biol 18:1244-1249

Lu HP, Xun L, Xie XS (1998) Single-molecule enzymatic dynamics. Science 282:1877-1882

Lukyanov KA, Chudakov DM, Lukyanov S, Verkhusha VV (2005) Innovation: photoactivatable fluorescent proteins. Nat Rev Mol Cell Biol 6:885-890

Marguet D, Lenne PF, Rigneault H, He HT (2006) Dynamics in the plasma membrane: how to combine fluidity and order. EMBO J 25:3446-3457

Matsunaga Y, Kidera A, Sugita Y (2015) Sequential data assimilation for single-molecule FRET photon-counting data. J Chem Phys 142:214115

McKinney SA, Joo C, Ha T (2006) Analysis of single-molecule FRET trajectories using hidden Markov modeling. Biophys J 91:19411951

Milles S, Koehler C, Gambin Y, Deniz AA, Lemke EA (2012) Intramolecular three-colour single pair FRET of intrinsically disordered proteins with increased dynamic range. Mol BioSyst 8:25312534

Morimatsu M, Takagi H, Ota KG, Iwamoto R, Yanagida T, Sako Y (2007) Multiple-state reactions between the epidermal growth factor 
receptor and Grb2 as observed using single-molecule analysis. Proc Natl Acad Sci U S A 104:18013-18018

Müller-Späth S, Soranno A, Hirschfeld V, Hofmann H, Rüegger S, Reymond L, Nettels D, Schuler B (2010) Charge interactions can dominate the dimensions of intrinsically disordered proteins. Proc Natl Acad Sci U S A 107:14609-14614

Murakoshi H, Iino R, Kobayashi T, Fujiwara T, Ohshima C, Yoshimura A, Kusumi A (2004) Single-molecule imaging analysis of Ras activation in living cells. Proc Natl Acad Sci U S A 101:7317-7322

Nakamura Y, Hibino K, Yanagida T, Sako Y (2016) Switching of the positive feedback for RAS activation by a concerted function of SOS membrane association domains. Biophys Physicobiol 13:1-11

Nguyen VT, Kamio Y, Higuchi H (2003) Single-molecule imaging of cooperative assembly of $\gamma$-hemolysin on erythrocyte membranes. EMBO J 22:4968-4979

Okamoto K, Sako Y (2012) Variational Bayes analysis of a photon-based hidden Markov model for single-molecule FRET trajectories. Biophys J 103:1315-1324

Okamoto K, Sako Y (2016) State transition analysis of spontaneous branch migration of the Holliday junction by photon-based singlemolecule fluorescence resonance energy transfer. Biophys Chem 209:21-27

Orrit M, Ha T, Sandoghdar V (2014) Single-molecule optical spectroscopy. Chem Soc Rev 43:973-976

Pack CG, Yukii H, Toh-e A, Kudo T, Tsuchiya H, Kaiho A, Sakata E, Murata S, Yokosawa H, Sako Y, Baumeister W, Tanaka K, Saeki Y (2014) Quantitative live-cell imaging reveals spatio-temporal dynamics and cytoplasmic assembly of the $26 \mathrm{~S}$ proteasome. Nat Commun 5:3396

Panyutin IG, Biswas I, Hsieh P (1995) A pivotal role for the structure of the Holliday junction in DNA branch migration. EMBO J 14:18191826

Persson F, Lindén M, Unoson C, Elf J (2013) Extracting intracellular diffusive states and transition rates from single-molecule tracking data. Nat Methods 10:265-269

Pirchi M, Tsukanov R, Khamis R, Tomov TE, Berger Y, Khara D, Volkov H, Haran G, Nir E (2016) Photon-by-photon hidden Markov model analysis for microsecond single-molecule FRET kinetics. J Phys Chem B 120:13065-13075

Rust MJ, Bates M, Zhuang X (2006) Sub-diffuraction-limit imaging by stochastic optical reconstruction microscopy (STORM). Nat Methods 3:393-795

Sako Y, Minoguchi S, Yanagida T (2000) Single molecule imaging of EGFR signal transduction on the living cell surface. Nat Cell Biol 2: $168-172$

Sako Y, Hiroshima M, Pack C-G, Okamoto K, Hibino K, Yamamoto A (2012) Live cell single-molecule detection in systems biology. WIREs Syst Biol Med 4:183-192

Sakon JJ, Weninger KR (2010) Detecting the conformation of individual proteins in live cells. Nat Methods 7:203-205

Sase I, Miyata H, Corrie JET, Craik JS, Kinosita K Jr (1995) Real time imaging of single fluorephores on moving actin with an epifluorescence microscope. Biophys J 69:323-328
Schuler B, Eaton WA (2008) Protein folding studied by single-molecule FRET. Curr Opin Struct Biol 18:16-26

Schuler B, Lipman EA, Eaton WA (2001) Probing the free-energy surface for protein folding with single-molecule fluorescence spectroscopy. Nature 419:743-748

Shütz GJ, Kada G, Pastuchenko VP, Schindler H (2000) Properties of lipid micordomains in a muscle cell membrane visualized by single molecule microscopy. EMBO J 19:892-901

Soranno A, Koenig I, Borgia MB, Hofmann H, Zosel F, Nettels D, Schuler B (2014) Single-molecule spectroscopy reveals polymer effects of disordered proteins in crowded environments. Proc Natl Acad Sci U S A 111:4875-4879

Sultana T, Takagi H, Morimatsu M, Teramoto H, Li CB, Sako Y, Komatsuzaki T (2013) Non-Markovian properties and multiscale hidden Markovian network buried in single molecule time series. J Cham Phys 139:245101

Talaga DS, Lau WL, Roder H, Tang J, Jia Y, DeGrado WF, Hochstrasser RM (2000) Dynamics and folding of single two-stranded coiled-coil peptides studied by fluorescent energy transfer confocal microscopy. Proc Natl Acad Sci U S A 97:13021-13026

Tokunaga M, Imamoto N, Sakata-Sogawa K (2008) Highly inclined thin illumination enables clear single-molecule imaging in cells. Nat Methods 5:159-161

Tomov TE, Tsukanov R, Masoud R, Liber M, Plavner N, Nir E (2012) Disentangling subpopulations in single-molecule FRET and ALEX experiments with photon distribution analysis. Biophys J 102:11631173

Ulbrich MH, Isacoff EY (2007) Subunit counting in membrane-bound proteins. Nat Methods 4:319-321

Viterbi A (1967) Error bounds for convolutional codes and an asymptotically optimum decoding algorithm. IEEE Trans Inf Theory 13:260 269

Webb SED, Roberts SK, Needham SR, Tynan CJ, Rolfe DJ, Winn MD, Clarke DT, Barraclough R, Martin-Fernandez M (2008) Singlemolecule imaging and fluorescence lifetime imaging microscopy show different structures for high- and low-affinity epidermal growth factor receptors in A431 cells. Biophys J 94:803-819

Wuttke R, Hofmann H, Nettels D, Borgia MB, Mittal J, Best RB, Schuler B (2014) Temperature-dependent solvation modulates the dimensions of disordered proteins. Proc Natl Acad Sci U S A 111:52135218

Xu K, Zhong G, Zhuang X (2013) Actin, spectrin, and associated proteins form a periodic cytoskeletal structure in axons. Science 339:452456

Xue HB, Jiao HJ, Liang JQ, Liu WM (2015) Non-Markovian full counting statistics in quantum dot molecules. Sci Rep 5:8978

Yoshizawa R, Umeki N, Yanagawa M, Murata M, Sako Y (2017) Singlemolecule fluorescence imaging of RalGDS on cell surfaces during signal transduction to Ras to Ral. Biophys Physicobiol 14:75-84

Zheng Y, Brown FLH (2004) Single molecule photon emission statistics for non-Markovian blinking models. J Chem Phys 121:3238 